\title{
Quantification of Mycelium of Botrytis spp. and the Antagonist Ulocladium atrum in Necrotic Leaf Tissue of Cyclamen and Lily by Fluorescence Microscopy and Image Analysis
}

\author{
G. J. T. Kessel, B. H. de Haas, C. H. Lombaers-van der Plas, E. M. J. Meijer, \\ F. M. Dewey, J. Goudriaan, W. van der Werf, and J. Köhl
}

\begin{abstract}
First, second, third, fourth, and eighth authors: DLO Research Institute for Plant Protection (IPO-DLO), P.O. Box 9060, 6700 GW Wageningen, the Netherlands; fifth author: Department of Plant Sciences, University of Oxford, South Parks Road, Oxford OX1 3RB, United Kingdom; and first, sixth, and seventh authors: Laboratory for Theoretical Production Ecology (TPE-WAU), P.O. Box 430, Wageningen Agricultural University, 6700 AK Wageningen, the Netherlands.
\end{abstract}

Accepted for publication 1 July 1999.

\begin{abstract}
Kessel, G. J. T., de Haas, B. H., Lombaers-van der Plas, C. H., Meijer, E. M. J., Dewey, F. M., Goudriaan, J., van der Werf, W., and Köhl, J. 1999. Quantification of mycelium of Botrytis spp. and the antagonist Ulocladium atrum in necrotic leaf tissue of cyclamen and lily by fluorescence microscopy and image analysis. Phytopathology 89:868-876.

A technique was developed to localize and quantify the internal mycelial colonization of necrotic leaf tissue of cyclamen (Cyclamen persicum) or lily (Lilium) by pathogenic Botrytis spp. and the antagonist Ulocladium atrum. This technique allows investigation of competitive substrate colonization by both fungi, which is a key process for biological control of Botrytis spp. by $U$. atrum. A combination of differential fluorescent label-

an antimouse fluorescein conjugate. Wheat germ agglutinin conjugated to the fluorochrome TRITC was used to label mycelium of both fungi. Image analysis was used to measure the relative surface area of the cryostat section covered by fluorescing hyphae of Botrytis spp. and by fluorescing hyphae of both fungi. A mathematical conversion was derived and used to calculate the relative mycelial volume of each fungal species in the necrotic tissue based on the measured relative surface areas. Temporal aspects of substrate colonization were studied in a short time series. An analysis of components of variance provided insight into spatial colonization patterns for the fungal species involved and allowed the design of efficient sampling strategies for future experiments.
\end{abstract} ing and image analysis was applied on cryostat sections of necrotic leaf tissue. Botrytis mycelium was labeled specifically by indirect immunofluorescence using a monoclonal antibody specific for Botrytis spp. and
Additional keywords: Botrytis cinerea, B. elliptica, interfungal competition, microbial ecology, sampling strategy.
Botrytis spp. cause economically important diseases in numerous greenhouse and field crops. In lilies (Lilium), B. elliptica can cause destructive leaf infections (leaf fire) that result in serious yield losses (11). In cyclamen (Cyclamen persicum), B. cinerea causes leaf rot and is considered to be one of the major pathogens in this crop. Currently, the control of Botrytis diseases depends on the frequent use of fungicides. However, Botrytis spp. have shown a great potential to develop fungicide resistance $(9,14)$. The threat of fungicide resistance and an increasing number of governmental restrictions on the use of pesticides has increased the need for alternative control measures. Biological control offers an environmentally friendly supplement or alternative to chemical control.

Necrotrophic pathogens such as Botrytis spp. kill plant tissue before invading it and sporulate exclusively on necrotic tissue. The saprophytic fungal antagonist Ulocladium atrum isolate 385 suppressed sporulation of Botrytis spp. in several necrotic plant tissues $(20,22,23)$. As demonstrated for Botrytis spp. in onion, reduction of sporulation of the pathogen caused a delay in disease buildup (21). The mechanism by which $U$. atrum suppresses sporulation of Botrytis spp. is unknown, but Köhl et al. (19) suggested that toxins and direct parasitism were not involved. Antagonism is probably due to nutrient competition.

Competitive substrate colonization by the pathogen and the saprophytic antagonist resulting in reduction of sporulation takes place

Corresponding author: G. J. T. Kessel; E-mail address: G.J.T.Kessel@IPO.DLO.NL

Publication no. P-1999-0816-01R

(C) 1999 The American Phytopathological Society within the necrotic plant tissue from the earliest phases of colonization onward. Detailed knowledge of the spatial and quantitative characteristics of the competitive colonization of necrotic plant tissue by these fungi is missing, and techniques to study this subject are, to the best of our knowledge, not available. Quantification of the spore-producing surface area $(12,22)$, conidial production (3), or fruiting body formation $(1,26)$ does not give information about the early stages of colonization or the extent of internal colonization of the tissue. Chemical techniques quantifying fungal constituents such as chitin or ergosterol $(16,24,30,31)$ are not very sensitive and cannot selectively quantify specific fungal populations (24). Immunological $(2,10,15,18,28,29)$ or molecular methods (17) can quantify the total (i.e., internal plus external) fungal biomass. Furthermore, chemical, immunological, and molecular techniques rely on homogenization of the colonized substrate, which makes them inappropriate to study spatial aspects of fungal colonization.

A thorough knowledge of competitive substrate colonization will result in identification and understanding of the possibilities and limitations of the antagonist. This knowledge is essential to predict when antagonist applications will result in successful disease control and when other control measures should be used.

The objective of this study was to develop a technique to quantify internal colonization of necrotic cyclamen tissue by $B$. cinerea and $U$. atrum and of necrotic lily tissue by $B$. elliptica and $U$. atrum. This was achieved using an immunohistological approach. Our method has the advantage of being able to provide both quantitative and qualitative data on two fungal species competitively colonizing necrotic plant tissue. 
Development of the procedure involved optimization of methods for sectioning necrotic cyclamen and lily tissue, optimization of dual fluorescent fungal labeling techniques, and development and optimization of an image analysis program. Variance components for the different sampling levels were estimated and used to design optimal sampling strategies for the experiments. As a final step, a mathematical conversion was derived that relates the measured relative surface area of the section covered by fungal hyphae to the volume of hyphae within the section as an estimator for fungal biomass.

\section{MATERIALS AND METHODS}

Cultures. $B$. cinerea isolate 700 originally was isolated from a diseased gerbera (Gerbera jamesonii). Isolate BE9401 of B. elliptica, isolated from a diseased lily, cv. Mont Blanc, was provided by E. van den Ende (Bulb Research Centre [LBO], Lisse, the Netherlands). U. atrum isolate 385 was isolated from a necrotic leaf tip of a field-grown onion (23). B. cinerea and B. elliptica were stored as conidial suspensions in $15 \%$ glycerol at $-80^{\circ} \mathrm{C}$. $U$. atrum was maintained on oatmeal agar slants (20 g of milled oats, $15 \mathrm{~g}$ of agar [Oxoid L13; Unipath Ltd., Basingstoke, United Kingdom], and 1 liter of tap water) at $4^{\circ} \mathrm{C}$. Before use, B. cinerea was cultured on oatmeal agar for 2 weeks at $20^{\circ} \mathrm{C}$ in the dark. B. elliptica was cultured on malt extract agar (Oxoid CM59) for 2 weeks at $18^{\circ} \mathrm{C}$ under the continuous combined light of an 18 -W black light and an 18-W cool-white fluorescent tube arranged $20 \mathrm{~cm}$ above the cultures. $U$. atrum was cultured on oatmeal agar for 4 weeks at $18^{\circ} \mathrm{C}$ in the dark. Conidial suspensions of all fungi were prepared by flooding the cultures with sterile tap water containing $0.01 \%$ Tween 80. After gentle rubbing with a rubber spatula to detach the conidia, suspensions were filtered through nylon gauze with a mesh of $200 \mu \mathrm{m}$. Conidial concentrations were determined using a hemacytometer and adjusted with sterile tap water containing $0.01 \%$ Tween 80 to $1 \times 10^{4}$ conidia per $\mathrm{ml}$ for $B$. elliptica and $1 \times 10^{6}$ conidia per $\mathrm{ml}$ for $B$. cinerea and $U$. atrum.

Plant material and leaf inoculation. Cyclamen plants $(C$. persicum) cv. Superserie were grown in a greenhouse set at $18^{\circ} \mathrm{C}$ during autumn without additional light. Flower buds were removed to prevent pollen deposition on the leaves, which is known to enhance infection by $B$. cinerea (5). Mature healthy leaves were removed from the plants and dried slowly at room temperature to simulate natural senescence as closely as possible (23). After drying, the petiole was removed, and the leaf was cut in half along the midvein and sterilized by gamma irradiation (4 Mrad, cobalt-60 gamma source; Gammaster BV, Ede, the Netherlands). Before inoculation, leaves were rehydrated in sterile tap water. Excess water was removed by blotting the leaves on dry sterile filter paper. Subsequently, each leaf was placed on top of two pieces of sterile filter paper (50 mm diameter) containing $0.6 \mathrm{ml}$ of sterile water in sterile plastic petri dishes $(55 \mathrm{~mm}$ diameter, one halved leaf per dish). Leaves in the petri dishes were sprayed with a conidial suspension of $B$. cinerea $\left(1 \times 10^{6}\right.$ conidia per $\left.\mathrm{ml}\right)$, a conidial suspension of $U$. atrum $\left(1 \times 10^{6}\right.$ conidia per $\left.\mathrm{ml}\right)$, conidial suspensions of both fungi at the same concentrations, or sterile tap water containing $0.01 \%$ Tween 80 using an atomizer (Desaga, Heidelberg, Germany). When appropriate, $U$. atrum was applied directly after the application of B. cinerea. This resulted in conidial densities of $26 \pm 8$ conidia per $\mathrm{mm}^{2}$ for $U$. atrum and $23 \pm 11$ conidia per $\mathrm{mm}^{2}$ for $B$. cinerea. Sprayed leaves were incubated at $18^{\circ} \mathrm{C}$ in the dark.

Asiatic hybrid lilies (Lilium) cv. Mont Blanc were grown in pots in the greenhouse at $18^{\circ} \mathrm{C}$ with $16 \mathrm{~h}$ of light per day. Flower buds were removed, as is usual in bulb production and to prevent pollen deposition on the leaves. Healthy leaves approximately $8 \mathrm{~cm}$ long were removed from 3-month-old plants, dried at $60^{\circ} \mathrm{C}$ for $48 \mathrm{~h}$, and sterilized by gamma irradiation (4 Mrad). Before inoculation, leaves were washed three times in sterile tap water to remove soluble nutrients. Excess water was removed by blotting the leaves on dry sterile filter paper. Subsequently, leaves were placed on top of two pieces of sterile filter paper ( $80 \mathrm{~mm}$ diameter) containing $1.5 \mathrm{ml}$ of sterile water in sterile plastic petri dishes $(90 \mathrm{~mm}$ diameter, four leaves per dish). Leaves in the petri dishes were sprayed with both a conidial suspension of $B$. elliptica $\left(1 \times 10^{4}\right.$ conidia per $\mathrm{ml})$ and a conidial suspension of $U$. atrum $\left(1 \times 10^{6}\right.$ conidia per $\left.\mathrm{ml}\right)$ or with sterile tap water containing $0.01 \%$ Tween 80 using an atomizer. U. atrum was applied $8 \mathrm{~h}$ after the application of B. elliptica. Four petri dishes were sprayed per treatment. Sprayed leaves were incubated at $18^{\circ} \mathrm{C}$ under the continuous combined light of an $18-\mathrm{W}$ black light and an 18-W cool-white fluorescent tube arranged $20 \mathrm{~cm}$ above the petri dishes.

Preparation, sectioning, and staining of colonized leaves. Lily or cyclamen leaf tissue samples approximately $3 \times 5 \mathrm{~mm}$ in size were cut using a scalpel and placed in an Eppendorf vial containing $1 \mathrm{ml}$ of $3 \%$ (wt/vol) paraformaldehyde in phosphate buffered saline (PBS) for overnight fixation. Samples from the same leaf were placed in the same Eppendorf vial. After fixation, leaf samples were transferred to a graded series of sucrose solutions with increasing concentrations of 5,10,20,30,40, and 50\% (wt/vol) in PBS. Samples were kept in each concentration for 0.5 to $1 \mathrm{~h}$. Samples were blotted on dry filter paper to remove excess sucrose solution before embedding in Tissue-Tek O.C.T. compound (Miles, Elkhart, IN). Cross sections, $8 \mu \mathrm{m}$ thick for cyclamen leaves and $16 \mu \mathrm{m}$ thick for lily leaves, were cut using a cryostat (Microm, HM 500 O; Microm Laborgeräte $\mathrm{GmbH}$, Walldorf, Germany) at $-30^{\circ} \mathrm{C}$. Tissue sections were transferred to poly-l-lysine (Sigma Chemical Co., St. Louis, MO; $0.1 \%$ in milliQ [wt/vol])-coated slides and dried on a slide drying bench at $50^{\circ} \mathrm{C}$ for $15 \mathrm{~min}$.

Hybridoma supernatant from cell line BC-KH4 (4) was used to selectively detect $B$. elliptica hyphae in lily tissue sections and $B$. cinerea in cyclamen tissue sections. The monoclonal antibody (MAb) $\mathrm{BC}-\mathrm{KH} 4$ was originally raised against a $B$. cinerea isolate from grapes and is considered to be genus specific (6). Wheat germ agglutinin conjugated to tetramethylrhodamine isothiocyanate (WGATRITC) was used to label both fungi in lily and cyclamen tissue sections. This lectin exhibits a specificity for hyphal fragments (25) and selectively binds to chitin.

Dual fluorescent labeling of hyphae in the tissue sections on the glass slides was carried out according to the following schedules. Slides with cyclamen tissue sections were rinsed in PBS containing $0.1 \%$ ( vol/vol) Tween 20 (PBST) for $10 \mathrm{~min}$ to remove the embedding material. Subsequently, $2 \%$ (wt/vol) bovine serum albumin (Art. 12018; Merck, Darmstadt, Germany) + 1\% (vol/vol) normal goat serum in PBS was applied for 30 min to block binding to nonspecific sites. The slides were then rinsed two times in PBST for $15 \mathrm{~min}$. The MAb BC-KH4 (diluted 1:5 in PBST $+0.1 \%$ [wt/vol] acetylated bovine serum albumin: PBS-BSA-C [Aurion, Wageningen, the Netherlands]) was then applied, and the slides were incubated overnight at $4^{\circ} \mathrm{C}$. After incubation, tissue sections were rinsed three times in PBST for $30 \mathrm{~min}$. FITC-conjugated $\mu$-specific goat-antimouse IgG (1020-02; Southern Biotechnology Associates, Birmingham, AL) (diluted 1:80 in PBST-BSA-C) and the nonspecific fluorescent stain WGA-TRITC (Sigma L-5266; $0.033 \mathrm{mg}$ of protein per $\mathrm{ml}$ of PBS-BSA-C) were mixed in equal volumes, applied to the sections, and incubated for $1 \mathrm{~h}$ at $20^{\circ} \mathrm{C}$. Sections were again rinsed three times in PBST, mounted in citifluor antiquench (glycerol in PBS; Citifluor Ltd., London), and covered with a coverslip. Treated samples were stored at $4^{\circ} \mathrm{C}$ in the dark for later examination.

Slides with lily tissue sections were rinsed in PBST for $10 \mathrm{~min}$ to remove the embedding material. Subsequently, a $0.3 \%$ casein (Sigma C-5890) solution in PBS was applied for 30 min to block binding to nonspecific sites. The slides were then rinsed twice in PBST for $5 \mathrm{~min}$ and twice in milliQ. The MAb BC-KH4 (diluted $1: 5$ in PBST $+0.1 \%$ [wt/vol] BSA:PBST-BSA) was then applied, and the slides were incubated overnight at $4^{\circ} \mathrm{C}$. Tissue sections were rinsed twice in PBST and twice in milliQ. FITC-conjugated goat- 
antimouse IgG (Sigma F-9006) (diluted 1:10 in PBST-BSA) and the nonspecific fluorescent stain WGA-TRITC (Sigma L-5266; $0.033 \mathrm{mg}$ of protein per $\mathrm{ml}$ of PBST-BSA) were mixed in equal volumes, applied to the sections, and incubated for $1 \mathrm{~h}$ at $20^{\circ} \mathrm{C}$. Sections were again rinsed twice in PBST and twice in milliQ and treated further as described for cyclamen tissue sections.

Differences in the staining procedure for cyclamen and lily tissue sections were due to higher levels of background fluorescence encountered in cyclamen tissue. Acetylated BSA (BSA-C) was used in the cyclamen protocol because it is highly efficient in suppressing charge-determined background fluorescence (34).

Microscopy. Stained tissue sections were examined with a Leica DM RB fluorescence microscope (Leica, St. Gallen, Switzerland) equipped with three filter sets housed in a rotating carrier. An HQ filter block (excitation 535/emission 610) was used for TRITC detection (Fig. 1A), an L4 filter block (BP450-490/RKP510/BP515-
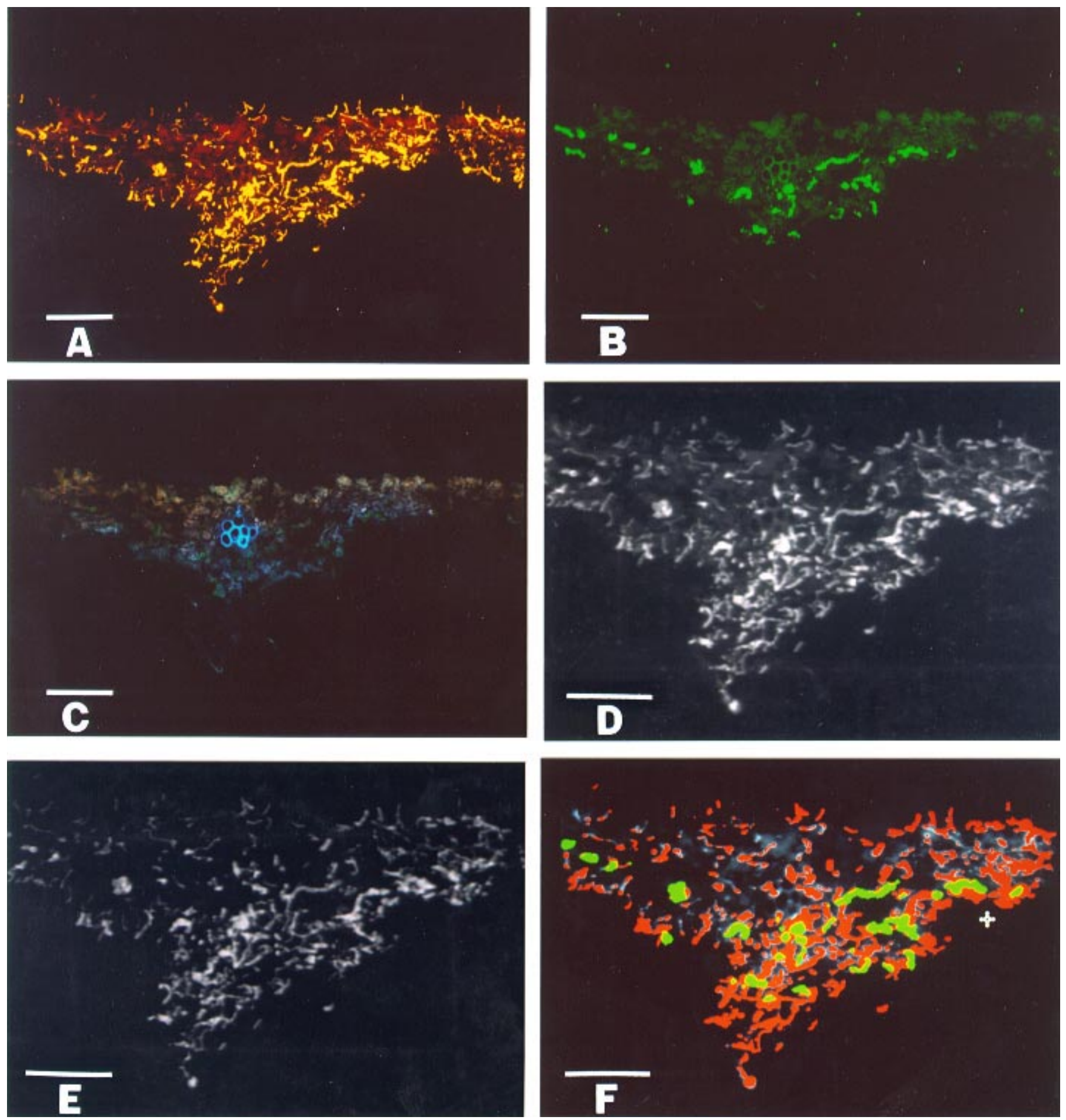

Fig. 1. A, B, and C, Photographs and D, E, and F, digitized images of a cross section of necrotic lily leaf colonized by Botrytis elliptica and antagonist Ulocladium atrum $51 \mathrm{~h}$ after inoculation. A, All fungi present in the section labeled using wheat germ agglutinin conjugated to fluorochrome TRITC. B, B. elliptica selectively labeled using the MAb BC-KH4 conjugated to fluorochrome FITC. C, Background image of the section as seen through a Leica A filter block, which does not show the fluorochromes TRITC and FITC. D, Gray-value image of A. E, Gray-value image calculated by subtracting gray-value image of C from $\mathbf{D}$, resulting in separation of fungal hyphae from original background present in D. F, Composite image with total fungal area detected (red) on top of image $\mathbf{E}$ and detected B. elliptica area (green) on top of both $\mathbf{E}$ and total fungal area detected. As a result of this layering, B. elliptica is represented by green areas, and $U$. atrum is represented by red areas. Bars represent $100 \mu \mathrm{m}$. 
560) was used for FITC detection (Fig. 1B), and an A filter block (BP340-380/RKP400/LP430) was used to obtain an image of the section based on autofluorescence only (Fig. 1C). Fungal hyphae were not visible with the A filter block. This filter did, however, provide a clear background image of the section.

Digitized images and photographs of the sections of colonized tissue were obtained at $\times 200$ magnification. Ektachrome ISO 100/21 ${ }^{\circ}$ film (Kodak, Rochester, NY) was used for the photographs.

Image analysis. The image analysis package MicroGop 2000S (Context Vision, Kista, Sweden) was used to automatically detect and estimate the surface area covered by each of the two dually labeled fungi. Using the tools of this package, a dedicated program was developed on a SPARC 5 workstation operating under Solaris 2.4 using X-WINDOWS as a graphical interface (SUN Microsystems, Inc., Mountain View, CA).

The images were obtained from a low-lux black and white cooled CCD camera PLT450 (resolution: $604 \times 576$ pixels; HCS Vision Technology, Son, the Netherlands) attached to the binocular tube of the Leica DM RB fluorescence microscope through a C-mount adapter and a $0.3 \times$ phototube. This camera produces low-noise images under poor light conditions (fluorescence) by means of integration techniques. An S-Bus frame grabber DASM-VIP (Analogic Corp., Peabody, MA) connected with the SPARC-Bus (SCSI) was used for acquisition of images.

The image analysis program consisted of a loop with a dialogue part for interaction with the user, gray scale image processing, thresholding, binary image processing, and display functions. This loop had to be completed for each section that was analyzed.

The loop started with the dialogue section in which the gray-value TRITC (Fig. 1D), FITC (data not shown), and background image (data not shown) were obtained using the HQ, L4, and A filter blocks, respectively. The user then draws a fine line along the edges of the section in the gray-value background image so that an accurate estimate for the surface area of the section can be obtained. The detected surface area of the section was contained in a binary image (data not shown).

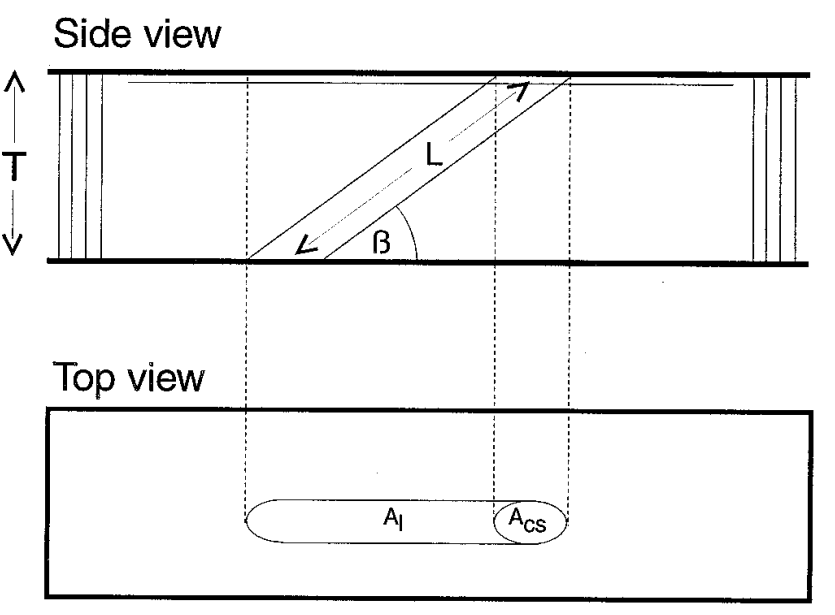

$A_{h}=A_{l}+A_{C S}$

Fig. 2. Schematic representation of a leaf tissue cross section containing one hyphal element. Side view of section shows a hyphal element of length $\mathrm{L}$ oriented in the section under angle $\beta$. T represents section thickness. Lower figure represents the section as it is seen through the microscope with the projected surface area of the hyphal element. According to the principle of Delesse (8), $A_{c s}$ is the correct estimator for relative volume of hyphal element in the section. For very thin sections $(T \approx 0)$, relative surface area of the projection of the hyphal element is $A_{h}=A_{c s}$. The second line from the top (thin) in the side view represents the lower border of such a section. For thicker sections, $A_{h}$ is composed of $A_{c s}$ plus relative surface area of the projection of the body of the hyphal element: $A_{h}=A_{l}+A_{c s}$. Thus, in thicker sections, $A_{h}$ overestimates the relative volume of the hyphal element by projected surface area of the body of the hyphae: $A_{l}$. Our calculation method for $V_{h}$ using formulas 1 and 2 corrects for this overestimation.
In the gray scale image processing step, the gray-value background image was subtracted from the gray-value TRITC (Fig. 1D) and gray-value FITC images, respectively. This resulted in corrected gray-value TRITC (Fig. 1E) and FITC (data not shown) images that almost exclusively contained the brightly fluorescing structures, representing the fungal hyphae, without the original background. The corrected gray-value TRITC and FITC images were thresholded, resulting in estimates for the fungal surface area contained in two binary images: a binary TRITC image (red overlay in Fig. 1F) and a binary FITC image (green overlay in Fig. 1F, which was placed on top of the red TRITC overlay). The threshold value was set manually in the first section of a batch of sections to assure that the detected fungal area represented, as closely as possible, the fungal area as it is seen through the microscope. The selected threshold values for FITC and TRITC detection were then stored and used for the whole batch of prepared sections. This procedure allowed a small correction for variation in labeling intensity between batches of stained sections.

In the binary image processing step, the surface area of the whole tissue section, the surface area of the tissue section covered by Botrytis spp., and the surface area of the section covered by both fungal species were measured from the binary background, binary FITC, and binary TRITC images, respectively $\left(\mu \mathrm{m}^{2}\right)$. The areal density (fraction of the section covered by hyphae [35]) was calculated from the measured values and converted into the relative volume, or volume density (35), of the mycelia of both species in the section using the procedure described below.

In the display procedure, Botrytis spp. and $U$. atrum hyphae were shown in two separate color overlays (green and red, respectively) on top of the gray-value TRITC image, thus showing how the Botrytis spp. and $U$. atrum hyphae were situated within the leaf (Fig. 1F). Values obtained for the relative surface area of $\mathrm{Bo}$ trytis, $U$. atrum, both species, and the area of the section were displayed and stored on file.

Mycelial volume. According to the principle of Delesse (8), the measured relative surface area or areal density of hyphal elements

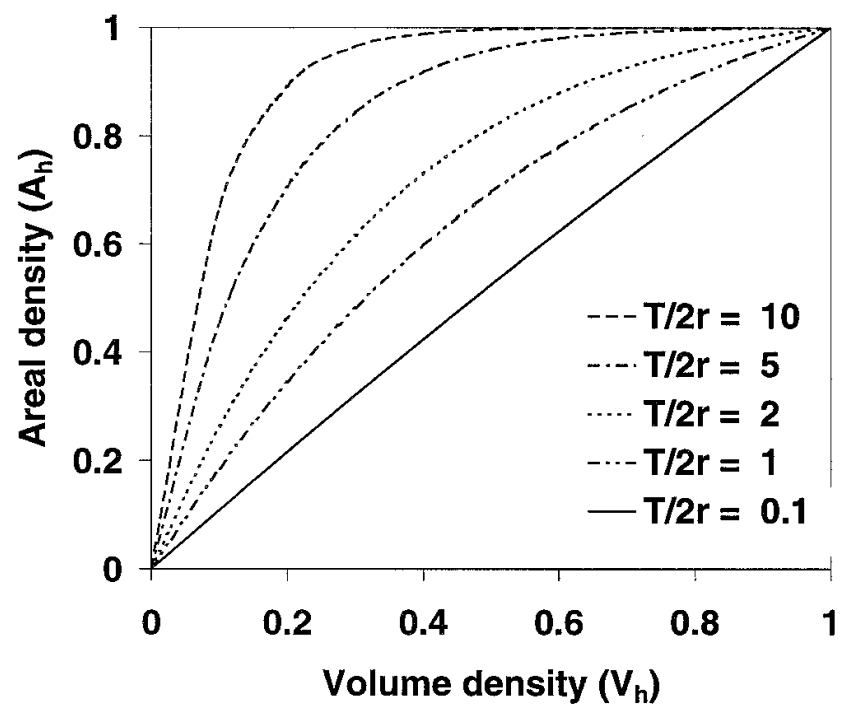

Fig. 3. Theoretical relationship between measured areal density $\left(A_{h}\right)$ and volume density $\left(V_{h}\right)$ of hyphal elements in sections according to formula 1 for different values of the ratio between section thickness and radius of the hyphal elements $(T /[2 r])$. Section thickness $(T)$ and hyphal radius $(r)$ determine contribution of the projection of the body of the hyphal element to measured areal density $\left(A_{h}\right)$ plus the chance of hyphal elements overlapping in the image used to measure surface density. The ratio $(T /[2 r])$, therefore, governs the theoretical relationship between areal density $\left(A_{h}\right)$ and volume density $\left(V_{h}\right)$. Volume densities of Ulocladium atrum and Botrytis cinerea in Table 2 were calculated using a section thickness of $8 \mu \mathrm{m}$. Hyphal radii for $U$. atrum and B. cinerea were 2.1 and $2.6 \mu \mathrm{m}$, respectively. This results in a $T /(2 r)$ ratio of approximately 2 . 
in sections $\left(A_{h}\right)$ is a good estimator for the relative volume or volume density of the hyphal elements $\left(V_{h}\right)$, provided the section is very thin. However, in our relatively thick sections, $A_{h}$ overestimates $V_{h}$ due to a significant contribution of the projection of the hyphal element to $A_{h}$ (Fig. 2).

The areal density of the Botrytis spp. in the section ( $A_{b}$, unitless) is measured using the Bc-KH4-FITC label, after which formula 1 is used to calculate $V_{b}$, the unitless volume density of the Botrytis spp. The total areal density ( $A_{h}$, unitless) is measured using the WGA-TRITC label. Substitution of $V_{b}$ and $A_{h}$ in formula 2 allows calculation of $V_{u}$ (unitless), the volume density of $U$. atrum. Both conversion formulas are based on established stereological principles. The complete derivation of formulas 1 and 2 is given in the appendix.

$$
\begin{gathered}
1-A_{b}=\left(1-V_{b}\right) e^{\left(-V_{b} \frac{T}{2 r_{b}}\right)} \\
1-A_{h}=\left(1-V_{b}-V_{u}\right) e^{\left(-V_{b} \frac{T}{2 r_{b}}-V_{u} \frac{T}{2 r_{u}}\right)}
\end{gathered}
$$

In formulas 1 and $2, A_{h}$ and $A_{b}$ represent the relative surface areas of the sections covered by the projection of hyphal elements of both species and the Botrytis spp., respectively, while $V_{b}$ and $V_{u}$ represent the relative volumes of the sections occupied by hyphal elements of the Botrytis spp. and U. atrum. T is the section thickness $(\mu \mathrm{m})$ and $r_{b}$ and $r_{u}$ represent the hyphal radius $(\mu \mathrm{m})$ of the Botrytis spp. and $U$. atrum. Thus, the relation between $A_{h}$ and $V_{h}$ is governed by the ratio between section thickness and the hyphal radii (Fig. 3). For known dimensions of the section, $V_{h}$ can easily be converted into the absolute volume of the hyphal elements in the sections.

Detection of Botrytis spp. and $\boldsymbol{U}$. atrum in tissue. Two experiments were carried out on sterile necrotic cyclamen leaves with $B$. cinerea and $U$. atrum, and one was carried out on sterile necrotic lily leaves with $B$. elliptica and $U$. atrum. The first cyclamen experiment was used to obtain estimates for the variance components at the different sampling levels of leaf, leaf sample, section within sample, and position within section. Variance components are indicative of the variability of fungal colonization between and within leaves and can be used to design optimal sampling strategies. Five sterile necrotic cyclamen leaves were inoculated with a conidial suspension of $B$. cinerea, and another five leaves were inoculated with a conidial suspension of $U$. atrum. Petri dishes were incubated at $18^{\circ} \mathrm{C}$ in the dark, arranged in a completely randomized design. After $36 \mathrm{~h}$ of incubation, four samples approximately $3 \times 5 \mathrm{~mm}$ in size were taken per leaf and prepared for sectioning. Per sample, two sets of approximately eight sections were collected, each set on a separate glass slide. The sets originated from locations 300 to $400 \mu \mathrm{m}$ apart. Sections within a set were cut in sequence. Tissue sections were stained using the nonspecific fluorescent stain (WGA-TRITC) only. One randomly chosen section per set was analyzed. Within sections, three positions were distin-

TABLE 1. Components of variance with standard errors in parentheses in-

\begin{tabular}{|c|c|c|}
\hline Source of variation ${ }^{\mathrm{a}}$ & Ulocladium atrum & Botrytis cinerea \\
\hline Leaves $\left(\sigma_{\text {leaf }}^{2}\right)$ & $5.5(4.7)$ & $5.6(4.8)$ \\
\hline Samples within leaves $\left(\sigma_{\text {sample }}^{2}\right)$ & $2.8(1.9)$ & $0.7(2.4)$ \\
\hline Sections within samples $\left(\sigma_{\text {section }}^{2}\right)$ & $0.9(1.7)$ & $1.3(3.2)$ \\
\hline Position within sections $\left(\sigma_{\text {position }}^{2}\right)$ & $3.8(1.2)$ & $8.3(2.7)$ \\
\hline
\end{tabular}
dicative of variability of fungal colonization between and within leaves

a The importance of variation between and within leaves as well as systematic differences between positions were examined by fitting a mixed model with position as explanatory variable and random effects for differences between leaves, between samples within leaves, and between sections within samples. Estimates of fixed effects of position and variance components were obtained using the method of residual maximum likelihood (REML) in Genstat 5. Systematic effects of position were assessed by the Wald statistic using the chi-square test. guished: the center of the section, and two positions left and right of the center near the edges of the section. Measurements were taken from three positions per sample, including a randomly chosen position in a section from the first set and from the two complementary positions in a section from the second set of a sample. Including measurements on all three positions per section would have doubled the number of measurements without a significant effect on the precision of the estimates. This resulted in a total of 120 measurements (two fungal species, five leaves per species, four samples per leaf, and three positions per sample).

For both fungi, the importance of variation between and within leaves, as well as systematic differences between positions, were examined by fitting a mixed model with position as the explanatory variable and random effects for differences between leaves, between samples within leaves, and between sections within samples. In the model, the random effects are assumed to be normally distributed, with mean zero and variances $\sigma_{\text {leaf }}^{2}, \sigma_{\text {samples within leaves, }}^{2}$ and $\sigma_{\text {section within sample, respectively. }}^{2}$

Estimates of fixed effects of position and variance components were obtained using the method of residual maximum likelihood (REML) in Genstat 5 (Numerical Algorithms Group Inc., Oxford, United Kingdom). Systematic effects of position were assessed by the Wald statistic using the chi-square test.

The second cyclamen experiment was designed to monitor colonization levels of the pathogen and the antagonist in a short time series. Sterile necrotic cyclamen leaves were sprayed with conidial suspensions of $B$. cinerea, $U$. atrum, both fungi, or sterile tap water $+0.01 \%$ Tween 80 and incubated at $18^{\circ} \mathrm{C}$ in the dark in a completely randomized design. Six replicate leaves per treatment and sampling time were included. Leaves were destructively sampled 0,30 , and $51 \mathrm{~h}$ after spraying. In accordance with the results of the analysis of components of variance, colonization levels were determined for the center position of one section of one sample for each leaf. Angular transformed colonization data for $U$. atrum and $B$. cinerea were subjected to analysis of variance (ANOVA) testing for effects of fungal inoculum, sampling time, and their interaction. In case $F$ tests were significant $(P<0.05)$, least significant difference tests $(P=0.05)$ were used for testing pairwise differences between the means.

In the lily experiment, sterile necrotic lily leaves were sprayed with conidial suspensions of B. elliptica and $U$. atrum or sterile tap water $+0.01 \%$ Tween 80 . $U$. atrum was applied $8 \mathrm{~h}$ after $B$. elliptica. Petri dishes were incubated at $18^{\circ} \mathrm{C}$ in the dark in a completely randomized design. Four replicate petri dishes with four leaves per dish were used per treatment. Colonization levels were determined 0,30 , and $51 \mathrm{~h}$ after application of the B. elliptica inoculum.

The hyphal diameter was measured in WGA-TRITC-labeled sections of both colonization experiments in sections sampled after 30 and $51 \mathrm{~h}$ of incubation. The hyphal radius, required for the conversion of areal densities of fungal colonization to the volume density (formulas 1 and 2), was calculated as the average of 24 measurements per species. B. cinerea, B. elliptica, and $U$. atrum were found to have hyphal radii of $2.6 \pm 0.3,2.7 \pm 0.1$, and $2.1 \pm 0.3 \mu \mathrm{m}$, respectively.

\section{RESULTS}

Immunofluorescent labeling and general lectin stain. The staining procedure resulted in a bright and consistent staining of hyphae of $B$. cinerea, B. elliptica, and $U$. atrum by the WGATRITC label (Fig. 1A) and of B. cinerea and B. elliptica hyphae by the MAb BC-KH4 + antimouse FITC conjugates (Fig. 1B). Hyphae of Botrytis spp. were visible with the TRITC- and FITCselective filters, whereas $U$. atrum hyphae were only visible with the TRITC-selective filter. Differences in staining intensity among B. cinerea, B. elliptica, and $U$. atrum for the WGA-TRITC stain were not observed. FITC-labeled hyphae in sections containing only 
$U$. atrum were never observed. Green or red fluorescing structures in sections of noninoculated leaves were not observed. This made it possible to distinguish the fungi from the background and to distinguish among the mycelia of the respective pathogens and the antagonist.

Detection threshold, quenching, and reproducibility. The detection program was applied on digitized images obtained from the CCD camera. Structures represented by one pixel were the smallest structures that could be detected. This corresponded to structures $1.2 \times 1.2 \mu \mathrm{m}$ in size at a $\times 200$ magnification. Smaller structures could be detected using higher magnifications.

Similarly to other fluorescent stains, the intensity of the stains described here was subject to quenching. However, with our staining procedure, the quenching rates were relatively low when compared with the quenching rate of fluorescein diacetate (FDA). When the measurements on the section in Figure 1 were repeated 7 months after the original measurements, during which time the sections were stored at $4^{\circ} \mathrm{C}$ in the dark, the relative surface areas detected were $4 \%$ for B. elliptica and $14 \%$ for $U$. atrum, compared with 5 and $17 \%$, respectively, in the original measurements.

When the section was exposed to continuous illumination using the HQ filter block for TRITC detection, the detected total surface area covered by fungal hyphae was reduced by $7 \%$ in 15 min and by $53 \%$ in 60 min (data not shown). This low quenching rate allowed ample time for measurements, which took about 1 min per sample. These results also indicate that it is possible to store nonexposed sections for at least several months at $4^{\circ} \mathrm{C}$ in the dark without significant loss of quality.

Reproducibility was tested by repeated measurements on the same section. For this purpose, a section of lily tissue colonized by $B$. elliptica and $U$. atrum after $51 \mathrm{~h}$ of incubation was used. Four replicate measurements resulted in almost identical results: $6.5 \pm 0.3 \%$ colonization by $B$. elliptica and $7.2 \pm 0.3 \%$ colonization by $U$. atrum. Small differences in the four images obtained from the section by the integrating CCD camera were the most likely cause for the differences that were found.

Variance components. Variance components for the different sampling levels of $B$. cinerea- or $U$. atrum-colonized necrotic cyclamen leaves are given in Table 1 . The calculated $P$ value of the chi-square test for the Wald statistic $(P=0.064)$ indicated that measurements near the edges of a section tend to be lower than measurements near the center of the section. This clear edge effect may be the result of over fixation of the exposed edges of a tissue sample by the paraformaldehyde, which is capable of blocking the binding sites for the MAb and the lectin through extensive crosslinking. This problem can be avoided by measuring at less-exposed locations near the center of the section.

For both fungi, the estimated components of variance in Table 1 suggest a large variation in colonization levels among leaves. The estimated components of variance from sources within the leaf suggest a spatially inhomogeneous colonization of leaves. Residual error variances generally were large, but because they are asymptotically estimated, their informative value is limited.

Based on the size of the estimated components of variance from the current experiment, the expected variance of the mean colonization level of a new experimental design can be calculated according to $\operatorname{var}_{\text {mean }}=\left(\sigma_{\text {leaf }}^{2} / n_{\text {leaf }}\right) \times\left\{1+1 / n_{\text {sample }} \times\left[\sigma_{\text {sample }}^{2} / \sigma_{\text {leaf }}^{2}+\right.\right.$ $\left.\left.\left(\sigma_{\text {section }}^{2} / \sigma_{\text {leaf }}^{2}\right) / n_{\text {section }}+\left(\sigma_{\text {position }}^{2} / \sigma_{\text {leaf }}^{2}\right) /\left(n_{\text {section }} \times n_{\text {position }}\right)\right]\right\}$.

Costs of labor and materials for the different steps in the sampling, processing, and measurement can be taken into account by introducing cost functions (32). Thus, the most efficient sampling scheme for each purpose can be designed. From the estimated components of variance, it is concluded that, in general, sampling schemes will be most efficient when the number of leaves is maximized with as little effort as possible at the lower sampling levels.

Temporal aspects of substrate colonization. As indicated by the significant effect of time on the $U$. atrum colonization level and the indication of significance for $B$. cinerea (Table 2), the vol- ume density of both fungi in the tissue increased during the entire incubation period. When B. cinerea or $U$. atrum was applied alone, the average volume density increased from 0 to 0.3 and $1.2 \%$ for $B$. cinerea and from 0 to 0.3 and $2.9 \%$ for $U$. atrum after 0,30 , and $51 \mathrm{~h}$ of incubation, respectively. When both fungi were applied in combination, both actively colonized the available tissue. The colonization levels reached after $51 \mathrm{~h}$ of competitive colonization were, however, lower than the levels reached during noncompetitive colonization (Table 2). This trend was only apparent after $51 \mathrm{~h}$ of incubation, accounting for the significant interaction between the effects of time and treatment on the $U$. atrum volume density. Apparently, $U$. atrum and B. cinerea are only hindering each other after the first phase of colonization. No fungal colonization was measured in leaf tissue sprayed with water containing $0.01 \%$ Tween 80 only (data not shown).

In comparison, the volume densities reached in sterile necrotic lily tissue by $B$. elliptica and $U$. atrum, applied in combination, increased from 0 to 0.4 and $1.3 \%$ for B. elliptica and from 0 to 0.8 and $3.1 \%$ for $U$. atrum after 0,30 , and $51 \mathrm{~h}$ of incubation, respectively. Similarly to the situation in cyclamen, both the pathogen and the antagonist colonized available tissue in the other's presence. Necrotic lily leaf tissue, however, supported higher fungal colonization levels than did necrotic cyclamen leaf tissue.

TABLE 2. Effect of fungal inoculation (treatment) and incubation period (time) on volume density of Ulocladium atrum and Botrytis cinerea, representing fungal colonization, in sterile necrotic cyclamen leaf tissue ${ }^{a}$

\begin{tabular}{|c|c|c|c|c|}
\hline & & & \multicolumn{2}{|c|}{ Average volume density (\%) } \\
\hline \multicolumn{3}{|c|}{ Comparison of means } & B. cinerea & U. atrum \\
\hline \multirow[t]{3}{*}{ Treatment } & B. cinerea & & $0.7^{\mathrm{b}}(0.73)$ & $0^{c}$ \\
\hline & U. atrum & & $0^{\mathrm{d}}$ & $1.6(1.58)$ \\
\hline & B. cinerea $+U$. atrum & & $0.3(0.27)$ & $0.4(0.43)$ \\
\hline \multirow[t]{3}{*}{ Time } & $0 \mathrm{~h}$ & & $0^{\mathrm{d}}$ & $0^{\mathrm{d}}$ \\
\hline & $30 \mathrm{~h}$ & & $0.3(0.26)$ & $0.2(0.23)$ \\
\hline & $51 \mathrm{~h}$ & & $0.7(0.74)$ & $1.8(1.78)$ \\
\hline \multirow[t]{9}{*}{ Treatment $*$ time } & B. cinerea & $0 \mathrm{~h}$ & $0^{\mathrm{d}}$ & $0^{\mathrm{d}}$ \\
\hline & & $30 \mathrm{~h}$ & $0.3(0.28)$ & $0^{\mathrm{c}}$ \\
\hline & & $51 \mathrm{~h}$ & $1.2(1.18)$ & $0^{c}$ \\
\hline & U. atrum & $0 \mathrm{~h}$ & $0^{\mathrm{d}}$ & $0^{\mathrm{d}}$ \\
\hline & & $30 \mathrm{~h}$ & $0^{\mathrm{d}}$ & $0.3(0.25)$ \\
\hline & & $51 \mathrm{~h}$ & $0^{\mathrm{d}}$ & $2.9(2.90)$ \\
\hline & B. cinerea $+U$. atrum & $0 \mathrm{~h}$ & $0^{\mathrm{d}}$ & $0^{\mathrm{d}}$ \\
\hline & & $30 \mathrm{~h}$ & $0.2(0.23)$ & $0.2(0.20)$ \\
\hline & & $51 \mathrm{~h}$ & $0.3(0.30)$ & $0.7(0.67)$ \\
\hline \multicolumn{3}{|l|}{$\mathrm{LSD}_{0.05}$} & & $(1.41)$ \\
\hline \multicolumn{3}{|l|}{ ANOVA $^{e}$} & \multicolumn{2}{|c|}{$F$ probability } \\
\hline \multicolumn{3}{|l|}{ Treatment } & 0.109 & 0.027 \\
\hline \multicolumn{3}{|l|}{ Time } & 0.098 & 0.004 \\
\hline \multicolumn{3}{|c|}{ Treatment $*$ time } & 0.150 & 0.033 \\
\hline
\end{tabular}

a Sterile necrotic cyclamen leaf tissue was homogeneously inoculated with conidial suspensions of $B$. cinerea, $U$. atrum, or a combination of both. Conidial concentrations were $1 \times 10^{6}$ conidia per $\mathrm{ml}$ for both species, resulting in conidial densities of $26 \pm 8$ and $23 \pm 11$ conidia per $\mathrm{mm}^{2}$ of leaf for $U$. atrum and $B$. cinerea, respectively. Inoculated tissue was destructively sampled after incubation in moist chambers at $18^{\circ} \mathrm{C}$ in the dark. Average volume densities (mycelial volume/volume of section [\%]) are based on one measurement in one section in each of six replicate leaves, except for the $U$. atrum volume density measured after $30 \mathrm{~h}$ of incubation in leaves inoculated with $U$. atrum only, which is based on one measurement in one section in each of four replicate leaves. The volume density was calculated from the measured areal density using conversion formulas 1 and 2 . Section thickness was $8 \mu \mathrm{m}$; hyphal radii for $U$. atrum and $B$. cinerea were 2.1 and $2.6 \mu \mathrm{m}$, respectively.

b Mean on normal scale and the corresponding mean on angular scale in brackets.

c Excluded because of negative values. B. cinerea mycelium was quantified using the FITC label and the TRITC label. Small differences between the images from both labels result in detection of small positive or negative quantities of $U$. atrum mycelium. Standard deviations were 0.2 and $0.3 \%$ after 30 and $51 \mathrm{~h}$ of incubation, respectively.

${ }^{\mathrm{d}}$ Excluded in the analysis of variance due to absence of variance in the observed data.

e Analysis of variance (ANOVA) and comparison of means on angular transformed volume densities per leaf. 


\section{DISCUSSION}

A technique was developed to localize and quantify the mycelium of Botrytis spp. and $U$. atrum within necrotic tissue. It provides both qualitative (visual) and quantitative information on the internal component of the fungal colonization of necrotic plant tissue.

The combination of a specific immunofluorescent label and a lectin conjugated to a fluorochrome is equivalent to the method reported by Pfender et al. (27), who used a dual-staining technique to observe antagonism of Pyrenophora tritici-repentis by the antagonistic fungus Limonomyces roseipellis in wheat straw. In their situation, however, quantification of mycelium was not possible, and the selectively stained fungus was not always visible with the nonselective stain. The latter difference might be caused by a difference in affinity for WGA-TRITC between the fungi used in both studies.

To obtain an accurate estimate for fungal colonization of the tissue section, it is necessary that the whole hypha is stained. In our case, both the chitin residues and the antigen to which the MAb $\mathrm{BC}-\mathrm{KH} 4$ binds are located throughout the fungal cell wall (6), ensuring that both stains visualize the full hyphae.

A common problem encountered when visualizing fungal hyphae within plant tissue using fluorescence microscopy is host autofluorescence, obscuring the fluorescence of the stained fungal hyphae. Several techniques have been applied to reduce host autofluorescence, including enzymatic degradation of stained host cellulose $(7,33)$ and counterstains $(27)$. During the current study, the extent of the autofluorescence problems was largely determined by the host species. Autofluorescence problems were minimized by the use of a specific MAb that did not cross-react with host tissues, a narrow band filter for the FITC detection, and the shading correction. For cyclamen tissue, it also was necessary to use acetylated BSA (BSA-C) and normal goat serum. BSA-C very effectively suppressed charge-determined background fluorescence (34), whereas the normal goat serum prevented aspecific binding of the (goatantimouse) FITC-conjugates. Autofluorescence countermeasures as applied by Pfender et al. (27), Trese and Loschke (33), or Daniel et al. (7) were not necessary.

For image analysis, it is necessary to separate the target structures from the rest of the image. This separation can be based on differences in gray value between the target and the background and/or structural characteristics of the target (e.g., length, width, shape). Overlapping hyphae, cut at different angles, result in a wide variety of possible shapes of fungal mycelium in the section. This makes it impossible to separate hyphae from the background by using structural characteristics. The detection was, therefore, based on differences in gray value between the fluorescing hyphae and the nonfluorescent background. Epifluorescence microscopy is especially suited for this purpose because it usually results in high contrasts. The higher the contrast between hyphae and background, the more accurate the detection will be. Use of the integrating CCD camera and image correction through the subtraction of a background image from the original gray-value FITC and TRITC images greatly enhanced the contrast between target structures and nontarget structures. However, weakly fluorescing hyphal structures might not be detected using this procedure. Our method is also sensitive to differences in staining intensity of the fungal hyphae within and between experiments. The background correction of the gray-value images and the manual adjustment of the detection threshold between experiments are tools used to overcome this problem. Nevertheless, the stains applied have to produce highcontrast images for the method to produce accurate results.

The $B$. cinerea areal density can be measured with both the general WGA-TRITC label and the specific Bc-KH4-FITC label. A measurement on the same section with each of the two labels will result in two nearly identical values for the $B$. cinerea areal density. Small differences between the measured values for each of the labels, however, result in small quantities of $U$. atrum detected because $U$. atrum colonization is calculated as the differ- ence between the surface area detected with the WGA-TRITC label and the surface area detected with the Bc-KH4 label. Although the average volume density of $U$. atrum detected in tissue inoculated with $B$. cinerea only is zero for all three sampling times, the bias in the detection of $U$. atrum is evident through the standard deviations of 0.2 and $0.3 \%$ after 30 and $51 \mathrm{~h}$ of incubation (Table 2). This problem is not relevant in situations in which it is known that only $B$. cinerea is present, but it influences the sensitivity of detection of $U$. atrum in situations in which both $U$. atrum and $B$. cinerea are present. The problem can be solved by using a specific label for both fungal species, making the indirect detection of one of the species obsolete.

The principle of Delesse (8), applied on leaf tissue sections colonized by fungi, states that the areal density of the cross sections through the hyphae $\left(A_{c s}\right.$ in Figure 2$)$ is a good estimator for the volume density of hyphal elements in the section. The areal density as measured using our technique would, therefore, be a good estimator for the volume density for very thin sections (Fig. 2). For thicker sections, conversion formulas 1 and 2 allow one to correctly estimate that the volume density, which in contrast to the areal density, is independent of section thickness. These formulas are based on the principle of Delesse (8) and the basic theories used to calculate the projected leaf area of crop canopies (13). The volume of hyphal elements in a section is an excellent estimator for fungal biomass. In addition, the conversion to volume allows a direct comparison of results obtained using sections of different thickness. This conversion therefore adds in the flexibility of this technique and facilitates a more accurate analysis of the generated data. The low values found for the areal densities of fungi in the sections belong to the first "linear" part of the curves in Figure 3, indicating that overlap in the projection of the hyphae hardly occurs.

Currently, a direct comparison between our technique and chemical, immunological, or molecular techniques is not possible because the other techniques do not distinguish between fungal species. A comparison with only one species would be inconclusive because our technique quantifies the internal component of fungal colonization, whereas the other techniques quantify total colonization: internal plus external mycelium, conidiophores, and conidia. The dynamics of the internal and external colonization are likely to be different. The internal, actively competing, mycelial component of fungal colonization is our prime interest when studying competitive substrate colonization by $U$. atrum and Botrytis spp.

Measurements on the colonization process of necrotic cyclamen and lily tissue by Botrytis spp. and $U$. atrum show that the species are capable of cocolonizing these tissues. However, growth tends to be reduced when both species are present compared with each species alone, especially during the second sampling interval. This observation, together with the observations by Köhl et al. (19) indicating that toxins are not involved in the interaction between Botrytis spp. and $U$. atrum, supports the hypothesis of nutrient competition as the mode of action. The analysis of variance components reveal that despite homogenous inoculation resulting in high and homogenous initial conidial densities on the leaf surfaces, the resulting colonization of necrotic tissue is not homogenous. Large differences are found in the colonization levels between and within leaves (Table 1). We believe that this variation reflects qualitative structural and nutritional differences between and within leaves with respect to colonization by $B$. cinerea and $U$. atrum.

A disadvantage of our technique is that it cannot distinguish between viable and nonviable mycelium. This disadvantage is shared with most other techniques for quantifying fungal colonization. This fact has to be included in the interpretation of experimental results, especially for the later phases of competitive substrate colonization.

The technique described in this paper quantifies the internal fungal colonization of necrotic tissue as a measure for the fungal biomass present internally in the tissue. It is capable of distinguishing between fungal species and can be applied in at least two host plants. The relatively simple principles underlying the quantifica- 
tion of fungal colonization as well as the basic nature of the image analysis tools used facilitate successful adaptation to other systems and environments. Currently, the availability of specific fungal labels seems to be the most limiting factor for a wider application. If specific antibodies for each of the two fungi studied were conjugated directly, each to a different fluorochrome, our technique could also be applied to field situations to quantify the mycelia of the target fungi in the presence of naturally occurring saprophytic fungal species.

Our technique generates a unique combination of quantitative and qualitative data on competitive substrate colonization, a key process in the biological control of Botrytis spp. by U. atrum. It is even sensitive enough to provide information on the potentially decisive early stages of colonization. We, therefore, conclude that it has the potential to provide new insights into fungal ecology essential for the development of new fungal biocontrol agents.

\section{APPENDIX}

To determine the volume density of hyphal elements in a leaf section, a stereological conversion formula was derived. The areal density of the hyphal elements in the section $\left(A_{h}\right.$; relative surface area of the projection of hyphal elements [35]) is measured using image analysis techniques. The volume density $\left(V_{h}\right.$; relative volume of hyphal elements in the section [35]) would, however, be a better estimator for the fungal biomass present in the section. According to the principle of Delesse (8), for very thin sections, $A_{h}=V_{h}$.

In very thin sections, $A_{h}$ is only constituted by $A_{c s}$, the relative surface area of the cross sections through the hyphae (Fig. 2). In thicker sections, however, $A_{h}$ consists of two components: $A_{c s}$ plus the projection of the body of the hyphal element in the section $\left(A_{l}\right)$

$$
A_{h}=A_{l}+A_{c s}
$$

Thus, $A_{c s}$ is the correct estimator for $V_{h}$. In thicker sections, the measured $A_{h}$ increasingly overestimates $V_{h}$ with an increasing section thickness.

To allow an accurate estimate of $V_{h}$, a mathematical expression for $A_{l}$ is derived and used to calculate $A_{c s}$, as an estimator for $V_{h}$, based on equation $1 \mathrm{~A}$.

Hyphae are considered to be cylindrical elements placed within the volume of a section. For each species, the separate elements are assumed to have an identical radius $(r)$ and to be oriented in all possible directions. The measured absolute area of the section is $A$; the calculated relative surface area of the hyphal elements in the section is $A_{h}$; and the known thickness of the section is $T$. The unknown total length of the hyphal elements in the section is $L$. The absolute volume of the section $(V)$ can be calculated as $V=A T$.

The true relative surface area of hyphal elements, in horizontal orientation and without overlap, would be $2 r L / A$.

Analogous to the calculation of the projected area of several leaf layers of a crop canopy (13), the relation between the measured relative surface area of the hyphal elements and the true relative surface area of the hyphal elements is given by

$$
A_{l}=1-e^{\left(-k \cdot \frac{2 \cdot r \cdot L}{A}\right)}
$$

The proportionality factor $k$ compensates for the different orientations of hyphal fragments, whereas the exponential function corrects for hyphae overlapping in projection. Assuming a spherical (or isotropical) distribution of the angle of the hyphal fragments with the horizon ( $\beta$ in Fig. 2 ), $k$ can be calculated as the ratio between the projected surface area of the hyphal fragments in a spherical distribution (without overlap) and the true surface area of the hyphal fragments (13): The projected surface area of hyphal elements, oriented in the section under an angle $\beta$, is calculated as $2 r L \cos (\beta)$ with a frequency distribution of $\beta$ that, in a spherical distribution, follows that of $\cos (\beta)$. The true surface area of the hyphal fragments is $2 r L$. Therefore

$$
k=\frac{\int_{0}^{1 / 2 \Pi} 2 r L \cos ^{2}(\beta) d(\beta)}{2 r L}=\frac{1 / 2 \Pi r L}{2 r L}=\frac{\Pi}{4}
$$

Equation $2 \mathrm{~A}$ combined with the result of $3 \mathrm{~A}$ gives the total relative projection of hyphal fragments with a spherical distribution

$$
A_{l}=1-e^{\left(-\Pi \cdot \frac{r \cdot L}{2 A}\right)}
$$

The relative volume of the hyphal elements in the section can be calculated as

$$
V_{h}=\frac{\Pi r^{2} L}{A T}
$$

Equations $4 \mathrm{~A}$ and $5 \mathrm{~A}$ can be combined into

$$
A_{l}=1-e^{\left(-V_{h} \frac{T}{2 r}\right)}
$$

$A_{l}$ will only contribute to $A_{h}$ when the projection of the body of the hyphal element is not overlapping with a cross section through a hyphal element $\left(A_{c s}\right) . A_{l}$ will, therefore, only contribute to $A_{h}$ for a fraction $\left(1-A_{c s}\right)=\left(1-V_{h}\right)$ of the available surface $A$. Thus, equation $6 \mathrm{~A}$ becomes

$$
A_{l}=\left(1-V_{h}\right)\left(1-e^{\left(-V_{h} \frac{T}{2 r}\right)}\right)
$$

When we substitute equation 7A into $1 \mathrm{~A}$ and substitute $A_{c s}$ by $V_{h}$, we get the desired relationship between the measured areal density $\left(A_{h}\right)$ of hyphal elements in a cross section and the volume density

$$
1-A_{h}=\left(1-V_{h}\right) e^{\left(-V_{h} \frac{T}{2 r}\right)}
$$

In equation $8 \mathrm{~A}, 1-A_{h}$ represents the relative surface area of the section not covered by the cross sections through fungal elements or projection of fungal elements, whereas $1-V_{h}$ represents the nonfungal relative volume in the section. $V_{h}$ cannot be written explicitly from equation $8 \mathrm{~A}$, but it can readily be solved numerically. Thus, the relation between the measured surface density $\left(A_{h}\right)$ and the hyphal volume density is governed by the ratio between the section thickness $(T)$ and the hyphal diameter $(2 r)$ of the fungal species under consideration (Fig. 3). Since $r$ can be measured and $T$ is known, the absolute hyphal volume in the section can also be calculated.

When both Botrytis spp. and $U$. atrum are present in the section, the total volume density $\left(V_{h}\right)$ is equal to the volume density of the Botrytis spp. $\left(V_{b}\right)$ plus the volume density of $U$. atrum $\left(V_{u}\right)$

$$
V_{h}=V_{b}+V_{u}
$$

The areal density of the Botrytis spp. in the section $\left(A_{b}\right)$ is measured specifically using the Bc-KH4-FITC label. In this case, $A_{h}=$ $A_{b}$ and equation $8 \mathrm{~A}$ can be converted into equation $10 \mathrm{~A}$, which is used to calculate the volume density of the Botrytis spp. in the section $\left(V_{b}\right) . r_{b}$ represents the hyphal radius of the Botrytis spp.

$$
1-A_{b}=\left(1-V_{b}\right) e^{\left(-V_{b} \frac{T}{2 r_{b}}\right)}
$$

For two fungal species, equation 10A can be extended to (combining equations $8 \mathrm{~A}$ and $9 \mathrm{~A}$ )

$$
1-A_{h}=\left(1-V_{b}-V_{u}\right) e^{\left(-V_{b} \frac{T}{2 r_{b}}-V_{u} \frac{T}{2 r_{u}}\right)}
$$

$A_{h}$ now represents the total areal density as it is measured using the WGA-TRITC label; $r_{b}$ and $r_{u}$ represent the respective hyphal 
radii of the Botrytis spp. and $U$. atrum. $V_{b}$ is calculated directly using equation $10 \mathrm{~A}$ and substituted into equation $11 \mathrm{~A}$ to calculate $V_{u}$.

\section{ACKNOWLEDGMENTS}

This work was supported by the Technology Foundation (STW, Utrecht, the Netherlands) through project WBI44.3284. We thank P. F. G. Vereijken, Centre for Biometry Wageningen (CBW), for statistical data analysis; N. J. Fokkema, DLO Research Institute for Plant Pathology (IPO-DLO), for stimulating discussions; and W. F. Pfender, USDA-ARS NFSPRC, Corvallis, OR, for advice on the nonspecific fluorescent fungal stain.

\section{LITERATURE CITED}

1. Adee, S. R., Pfender, W. F., and Hartnett, D. C. 1990. Competition between Pyrenophora tritici-repentis and Septoria nodorum in the wheat leaf as measured with de Wit replacement series. Phytopathology 80: $1177-1182$.

2. Bermingham, S., Dewey, F. M., and Maltby, L. 1995. Development of a monoclonal antibody-based immunoassay for the detection and quantification of Anguillospora longissima colonizing leaf material. Appl. Environ. Microbiol. 61:2606-2613.

3. Biles, C. L., and Hill, J. P. 1988. Effect of Trichoderma harzianum on sporulation of Cochliobolus sativus on excised wheat seedling leaves. Phytopathology 78:656-659.

4. Bossi, R., and Dewey, F. M. 1992. Development of a monoclonal antibody-based immunodetection assay for Botrytis cinerea. Plant Pathol. 41:472-482.

5. Chou, M. C., and Preece, T. F. 1968. The effect of pollen grains on infections caused by Botrytis cinerea Fr. Ann. Appl. Biol. 62:11-22.

6. Cole, L., Dewey, F. M., and Hawes, C. R. 1996. Infection mechanisms of Botrytis species: Pre-penetration and pre-infection processes of dry and wet conidia. Mycol. Res. 100(3):277-286.

7. Daniel, O., Schönholzer, F., and Zeyer, J. 1995. Quantification of fungal hyphae in leaves of deciduous trees by automated image analysis. Appl. Environ. Microbiol. 61:3910-3918.

8. Delesse, M. A. 1847. Procédé mécanique pour déterminer la composition des roches. C. R. Acad. Sci. (Paris) 25:544-545.

9. de Waard, M. A., Georgopoulos, S. G., Hollomon, D. W., Ihsii, H., Leroux, P., Ragsdale, N. N., and Schwinn, F. J. 1993. Chemical control of plant diseases: Problems and prospects. Annu. Rev. Phytopathol. 31:403-421.

10. Dewey, F. M., Twiddy, D. R., Phillips, S. I., Grose, M. J., and Wareing, P. W. 1992. Development of a quantitative monoclonal antibody-based immunoassay for Humicola lanuginosa on rice grains and comparison with conventional assays. Food Agric. Immunol. 4:153-167.

11. Doss, R. P., Chastagner, G. A., and Riley, K. L. 1986. Screening ornamental lilies for resistance to Botrytis elliptica. Sci. Hortic. 30:237-246.

12. Elad, Y., Köhl, J., and Fokkema, N. J. 1994. Control of infection and sporulation of Botrytis cinerea on bean and tomato by saprophytic yeasts. Phytopathology 84:1193-1200.

13. Goudriaan, J., and van Laar, H. H. 1994. Modelling Potential Crop Growth Processes. Pages 95-110 in: Current Issues in Production Ecology. Vol. 2. Kluwer Academic Publishers, Dordrecht, the Netherlands.

14. Gullino, M. L. 1992. Chemical control of Botrytis spp. Pages 217-222 in: Recent Advances in Botrytis Research. K. Verhoeff, N. E. Malathrakis, and B. Williamson, eds. Pudoc Scientific Publishers, Wageningen, the Netherlands.

15. Harrison, J. G., Barker, H., Lowe, R., and Rees, E. A. 1990. Estimation of amounts of Phytophthora infestans by enzyme-linked immunosorbent assay. Plant Pathol. 39:274-277.

16. Hicks, R. E., and Newell, S. Y. 1983. An improved gas chromatographic method for measuring glucosamine and muramic acid concentrations.
Anal. Biochem. 128:438-445.

17. Hu, J., Nazar, R. N., and Robb, J. 1993. Quantification of Verticillium biomass in wilt disease development. Physiol. Mol. Plant Pathol. 42:23-26.

18. Karpovich-Tate, N., Spanu, P., and Dewey, F. M. 1998. Use of monoclonal antibodies to determine biomass of Cladosporium fulvum in infected tomato leaves. Mol. Plant-Microbe Interact. 11:710-716.

19. Köhl, J., Bélanger, R. R., and Fokkema, N. J. 1997. Interaction of four antagonistic fungi with Botrytis aclada in dead onion leaves: A comparative microscopic and ultrastructural study. Phytopathology 87:634-642.

20. Köhl, J., Gerlagh, M., De Haas, B. H., and Krijger, M. C. 1998. Biological control of Botrytis cinerea in cyclamen with Ulocladium atrum and Gliocladium roseum under commercial growing conditions. Phytopathology 88:568-575.

21. Köhl, J., Molhoek, W. M. L., van der Plas, C. H., and Fokkema, N. J. 1995. Suppression of sporulation of Botrytis spp. as a valid biocontrol strategy. Eur. J. Plant Pathol. 101:251-259.

22. Köhl, J., Molhoek, W. M. L., van der Plas, C. H., and Fokkema, N. J. 1995. Effect of Ulocladium atrum and other antagonists on sporulation of Botrytis cinerea on dead lily leaves exposed to field conditions. Phytopathology 85:393-401.

23. Köhl, J., van der Plas, C. H., Molhoek, W. M. L., and Fokkema, N. J. 1995. Effect of interrupted leaf wetness periods on suppression of sporulation of Botrytis allii and Botrytis cinerea by antagonists on dead onion leaves. Eur. J. Plant Pathol. 101:627-637.

24. Lumsden, R. D., Carter, J. P., Whipps, J. M., and Lynch, J. M. 1990. Comparison of biomass and viable propagule measurements in the antagonism of Trichoderma harzianum against Pythium ultimum. Soil Biol. Biochem. 22:187-194.

25. Morrell, J. J., Gibson, D. G., and Krahmer, R. L. 1985. Effect of fluorescent-labeled lectins on visualization of decay fungi in wood sections. Phytopathology 75:329-332.

26. Newton, M. R., Kinkel, L. L., and Leonard, K. J. 1997. Competition and density-dependent fitness in a plant parasitic fungus. Ecology 78(6): 1774-1784.

27. Pfender, W. F., King, L. G., and Rabe, J. R. 1991. Use of dual-stain fluorescence microscopy to observe antagonism of Pyrenophora tritici-repentis by Limonomyces roseipellis in wheat straw. Phytopathology 81 : 109-112.

28. Plasencia, J., Jemmerson, R., and Banttari, E. E. 1996. Production and characterization of monoclonal antibodies to Verticillium dahliae and development of a quantitative immunoassay for fungal biomass. Phytopathology 86:170-176.

29. Ricker, R. W., Marois, J. J., Dlott, J. W., Bostock, R. M., and Morrison, J. C. 1991. Immunodetection and quantification of Botrytis cinerea on harvested wine grapes. Phytopathology 81:404-411.

30. Ride, J. P., and Drysdale, R. D. 1972. A rapid method for chemical estimation of filamentous fungi in plant tissue. Physiol. Plant Pathol. 2:7-15.

31. Seitz, L. M., Sauer, D. B., Burroughs, R., Mohr, H. E., and Hubbard, J. D. 1979. Ergosterol as a measure of fungal growth. Phytopathology 69: 1202-1203.

32. Sokal, R. R., and Rohlf, F. J. 1995. Pages 309-320 in: Biometry: The Principles and Practice of Statistics in Biological Research. 3rd ed. W. H. Freeman and Co., New York.

33. Trese, A. T., and Loschke, D. C. 1990. High contrast resolution of the mycelia of pathogenic fungi in corn tissue after staining with calcofluor and destaining with cellulase. Phytopathology 80:196-200.

34. van de Plas, P., and Leunissen, J. L. M. 1993. Ultrasmall gold probes: Characteristics and use in immuno(cyto)chemical studies. Pages 241-257 in: Methods in Cell Biology. Vol. 37, Antibodies in Cell Biology. J. D. Asai, ed. Academic Press, London.

35. Weibel, E. R. 1979. Stereological Methods. Vol. 1, Practical Methods for Biological Morphometry. Academic Press, London. 